\title{
The Ejection of Brown Dwarfs from Unstable Multiples
}

\author{
Eduardo Delgado-Donate \& Cathie Clarke \\ Institute of Astronomy, University of Cambridge, Madingley Road, \\ Cambridge CB3 OHA, U.K.
}

\begin{abstract}
We report on high-resolution hydrodynamic simulations of small stellar clusters that explore the effects of substructure in starforming regions. These calculations, when applied to the substellar regime, provide clear evidence for dynamical interactions to play a key role as a formation mechanism for brown dwarfs. Several observational consequences of this process are discussed.
\end{abstract}

\section{Introduction}

We have performed a large number of high-resolution hydrodynamic simulations of small clusters, still in the embedded phase, in which it is explored the consequences that substructure in star-forming regions may have on the properties of the resulting stars and brown dwarfs (see Delgado-Donate et al. 2002 for details). Each simulation consists of an isothermal spherical gas cloud with 5 steilar equal-mass point masses (which act as stellar seeds) in its interior. The initial mass of each cluster is a scale-free quantity.

As a simulation proceeds, gas is accreted by the protostars (initially only $10 \%$ of the mass is in the stellar seeds) and close encounters take place, which produce the ejection of some of the objects, invariably the lower-mass members of the cluster. The final mass of each star is determined by two processes: first, the seeds compete for the gas reservoir as the cloud collapses and strong dynamical interactions occur. This results into a bimodal pattern of mass acquisition: the central binary system accretes most of the mass while the ejected objects and outer companions acquire very little. Secondly, the initial mass of the cloud is set, e.g. from a core mass probability distribution (CMF). In this paper we have chosen a power-law CMF of slope -2.35 (Salpeter IMF) in the range of core masses 0.25 to $10 \mathrm{M}_{\odot}$, where this choice has been motivated by the results of Motte et al. (1998).

The calculations show that the population of escapers and outer companions is abundant in brown dwarfs. Some of their properties are sketched below.

\section{Results}

Fig. 1 shows the mass ratio of multiple systems in terms of the mass of the primary component. It can be readily seen that brown dwarfs produced by dynamical interactions are rare as binary companions. On the other hand, among hierarchical triples and quadruples the outermost object is very often a brown 


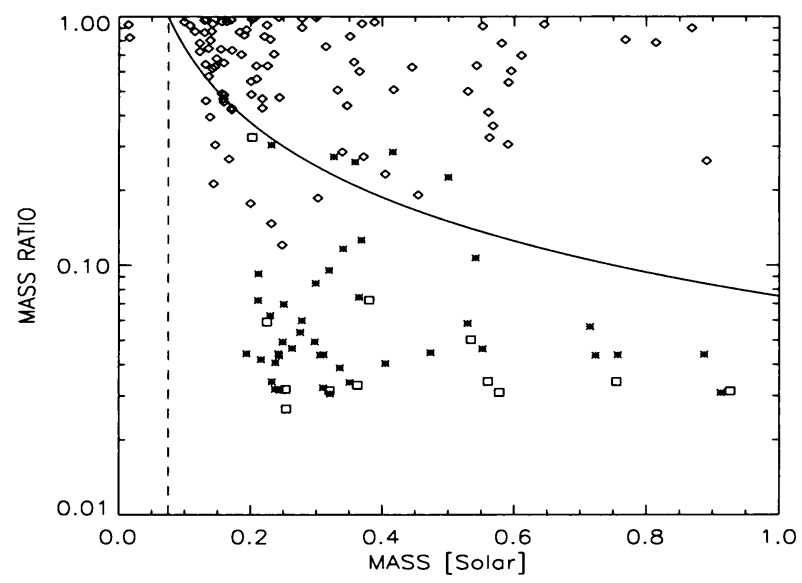

Figure 1. Mass ratio versus primary mass. (Diamonds=binaries, asterisks =triples, and squares=quadruples). To the left of the dashed line: binaries composed only of brown dwarfs; in between the lines: pairs with a brown dwarf companion; to the right of the solid line: binaries made up of normal stars.

dwarf, with a probability higher than $90 \%$ for low-mass primaries. In this sense, no brown dwarf desert at wide separations should be apparent if the inner components were not resolved (see Gizis et al. 2001). Brown dwarfs can be wide binary companions, but in this case the primary is predicted to be a binary system. Binary brown dwarfs are equally improbable in this scenario, with the exception of some scarce tight, nearly equal mass pairs.

The break-up of these small clusters imprint a clear kinematic signature on the objects involved. Binary systems attain speeds that are $\sim 10$ times lower than the typical ejection speed of single stars. Although there is no appreciable dependence of the final velocity of the singles on mass, brown dwarfs acquire as a class higher velocities than stellar objects. With velocities of a few $\mathrm{km} \mathrm{s}^{-1}$, it is possible that ejected brown dwarfs would be detectable in the vicinity of Class O objects, as suggested by Reipurth \& Clarke (2001). Relatively few brown dwarfs have velocities in excess of $5 \mathrm{~km} \mathrm{~s}^{-1}$, however, so that the bulk of brown dwarfs in the Pleiades would have been retained, given that the escape velocity in the young Pleiades is likely to have exceeded this value. On the other hand, smaller stellar associations such as Taurus could have lost a significant fraction of its substellar population.

\section{References}

Delgado-Donate E.J., Clarke C.J. \& Bate M.R., 2002, MNRAS, submitted

Gizis, J., et al. 2001. ApJ, 551, L163

Motte, F., Andre, P. \& Neri, R., 1998. A\&A, 336,150

Reipurth, B. \& Clarke, C., 2001. AJ, 122,432 